\title{
2. Principles of sustainability science
}

Over the past twenty years, an increasing number of researchers, practitioners and science policy officials have become engaged in sustainability science. This trend reflects the growing concerns amongst politicians, entrepreneurs and the public at large about the failure of science to provide operational solutions for addressing the sustainability challenges discussed above. More recently, the growing interest in sustainability science has been triggered by visible phenomena such as increasing oil and food prices, global warming and the continuing disappearance of species and biodiversity rich ecosystems. As many observers have mentioned, sustainability science is not, however, a scientific discipline by any usual definition (Rapport, 2007; Perrings, 2007). Rather it is a research field characterized by a new form of collaboration amongst disciplines and between disciplines and sustainability stakeholders. In a special issue of the Proceedings of the US National Academy of Science, Elinor Ostrom (2007) noted that, if sustainability science is to grow into a mature field of research, we must use the knowledge acquired in the separate disciplines of anthropology, biology, ecology, economics, environmental science, geography, history, law, political science, psychology and sociology to build and strengthen the diagnostic and analytical capabilities of the stakeholders who are directly confronted with practical sustainability problems (Ostrom et al., 2007).

The primary focus of sustainability science is to achieve the policy goal of sustainability, which encompasses ecological, economic, social, cultural and governance dimensions (Patterson and Glavovic, 2013). It is both an interdisciplinary and a transdisciplinary field of research - combining scientific and non-scientific expertise (see section 2.3 below) - that seeks to understand the complexities of coupled socio-ecological systems and develop practical solutions that promote ecological, economic and social sustainability.

Clearly sustainability science is still a relatively young field of research, with initially, at least, partly a different focus of research in Europe, Japan and the USA. As noted in the overview by Jaeger (2011), European practitioners have initially moved towards participatory, iterative processes with an implementation orientation, while Japan started with a technologybased approach and has only recently begun to pay more attention to the 
human dimensions, and the USA has prioritized interdisciplinary research on complex socio-ecological systems. However, despite these initial differences in approach, the discussions and projects in the scientific community over the past decades have clarified the common characteristics of sustainability science. In particular, recent discussions in the journals Sustainability Science and Ecological Economics characterize the agenda of the research field of sustainability science according to three core research dimensions (Wiek et al., 2012; Baumgaertner and Quaas, 2010):

1. sustainability science has to address the question of how coupled socio-ecological systems have evolved (past), are currently functioning (present), and might further develop (future), in order to identify the key sustainability problems to be addressed;

2 . in the context of this understanding of the sustainability challenges, sustainability science has to specify what are the ethical objectives of sustainability to be attained by taking into account the intrinsic limits of the exploitation of the earth's resources and how coupled socio-ecological systems would function and look in compliance with a variety of value-laden goals and objectives; and

3 . sustainability science has to explore with social actors and practitioners which transition pathways are viable for coupled socio-ecological systems and what strategies can be adopted to find solutions to the sustainability problems.

As can be seen from these three core dimensions, sustainability science combines a descriptive-analytical perspective on coupled socio-ecological systems, with a transformational** agenda, within an explicitly ethical perspective on strong sustainability and an engagement with social actors and practitioners. Because of this focus on a transformational agenda, and the aim of bridging the gap between science and society, some scholars have qualified sustainability science as an applied science (Clark and Dickson, 2003). However, such a perspective clearly misses the close interrelationships between the ethical perspective on sustainability, the need for innovative theoretical approaches to coupled socio-ecological systems and the transformational agenda, as can be seen in the need to rethink approaches in political sciences, economics and psychology inter alia to address the sustainability issues (Brousseau et al., 2012a; 2012b). Moreover, as highlighted in the report of the MASIS expert group on "Challenging futures of science in society", prepared for the Directorate General Research of the European Union (European Commission, 2009), such a combination of descriptive-analytical perspectives and transformational ethical and stakeholder analysis is not unusual in scientific research. Indeed, as 
clearly stated, the contrast between formal hypothetic-deductive scientific research on the one hand (both basic and applied) and socially relevant research (to specific context and value-laden goals and objectives) on the other hand is not a contrast of principles (European Commission, 2009 , p. 12). The contrast has more to do with the institutional division of labour than with the nature of scientific research. The combination of scientifically grounded and socially relevant research occurs again and again in history and in present-day science (see Stokes, 1997; Rip, 1997). This combination is not present in all disciplines and scientific fields in the same way, but as can be seen from the current debate on sustainability, it is clearly a defining feature of sustainability science.

Institutionally, a good indicator of the increasing importance of such research programmes combining conventional scientific excellence with social relevance is the spread of transdisciplinary research centres in various fields of research beyond sustainability science. The US Engineering Research Centres, the UK interdisciplinary research centres, and the Australian Collaborative Research Centres all started in the 1980s, and by now such centres have been established throughout Europe (European Commission, 2009, p. 13). In the Netherlands, in the Scandinavian countries and in Germany (for example through the Fraunhofer Institutes) large parts of public research funding are currently dedicated to such interdisciplinary and transdisciplinary research. Sustainability science is in that respect still a newcomer, but potentially this emerging field will become a very important member of the group of directly socially relevant research programmes, given the challenges reviewed above.

\subsection{STRONG SUSTAINABILITY AS THE NORMATIVE FOUNDATION OF SUSTAINABILITY SCIENCE}

Sustainability has become part of the mainstream policy discourse over the last two decades. However, in practice, policy objectives related to sustainability are often very modest, especially because of the still widespread belief in the possibility of decoupling (in spite of the growing evidence against the possibility of a general decoupling of economic growth and the increasing use of natural resources; see Chapter 1 above) and the tension with the dominant model of a consumption driven, low interest rate, economy. Therefore it is important to go beyond lip-service to the notion of sustainability and to specify its meaning as it emerges from the contemporary debates in environmental ethics and theories of justice. 


\subsubsection{Defining the Ethics of Strong Sustainability}

In general terms, sustainability aims at justice in the domain of socioecological relationships and in view of the long-term and inherently uncertain future, including both justice between humans of different generations (intergenerational justice) and justice between different humans of the same generation (intra-generational justice) (Baumgaertner and Quaas, 2010). These aspects are, for example, expressed in the widely accepted definition given by the Brundtland commission of the United Nations in 1987 (WCED, 1987, p. 43):

Sustainable development is development that meets the needs of the present without compromising the ability of future generations to meet their own needs. It contains within it two key concepts: the concept of "needs". In particular the essential needs of the world's poor, to which overriding priority should be given; and the idea of limitations imposed by the state of technology and social organisation on the environment's ability to meet present and future needs.

However, this conventional definition of sustainability is still very abstract. Indeed, it only says that, from a long-term perspective on socio-ecological relationships, members of the present generation have "something" that other members of the present generation and members of future generations need, in order to satisfy their own needs, and that we therefore need to preserve in a satisfactory manner. But what is that "something"?

Some have argued that we need to transmit a certain level of economic welfare to future generations. Future generations should, in principle, have a similar or even higher level of welfare than the present generation. However, such a vision does not seem to be defensible (Claassen, 2011, p. 204). First, economic welfare does not necessarily lead to a better satisfaction of our aspirations in life or a more just society (see the discussions on GDP in section 3.2.1 below). Second, even if "well-being" instead of welfare would be used as a measure of our aspirations in life, such well-being still cannot be transmitted directly to future generations. The well-being of future generations will be determined by circumstances that we cannot influence now. Third, future generations have their own moral autonomy and will make their own choices. A better understanding of sustainability therefore is that we should aim to preserve the possibility of all present and future generations to make their own choices in their aspiration to an accomplished and just life.

Irrespective of our particular understanding of intergenerational ethics, what needs to be transmitted cannot be fully captured in terms of current levels of capital. Preserving or extending the actual capabilities for 
self-determination of future persons is just as important (Sen, 1999). In a "capabilities approach", well-being cannot be reduced to individual utility; it necessarily implies a reasoned judgement on what is valuable and worth achieving, as well as the real capability to act in order to achieve it.

The possibility of other members of the present generation, and of future generations, to acquire a certain level of autonomy of choice can be understood in terms of a combination of the two types of capital discussed in section 1.1: natural capital and human capital (Claassen, 2011, p. 204). Human capital includes produced/technological capital, such as technological artefacts and products of labour, cultural capital, social capital and institutions. Natural capital includes both living and non-living natural resources, and the ecosystems and ecosystem services provided by these. As discussed above, current analysis shows that it is an illusion to believe that in transmitting the necessary level of human capital to future generations, technology will allow us to substitute all natural capital by one or another form of technological/produced capital, while preserving the same level of choice. Therefore, to preserve the capability of the present and future generations to make their own choices, efforts have to be made to keep certain forms of natural capital intact. In short, there is the need to adopt a strong sustainability perspective for the natural resources that are critical to maintain these possibilities of choice in the short and long-term future.

\subsubsection{The Task of Operationalizing the Ethical Framework}

The current debate on sustainability clearly leads to a growing consensus amongst policy makers and scientists that preserving the capability of choice of present and future generations implies a duty to preserve certain critical forms of natural capital (Claassen, 2011). However, this does not allow policy makers and scientists to close the debate on the meaning of sustainability, nor will it lead them to adopt a single and uniform definition of the practical objectives to be agreed upon for reaching sustainability. Indeed, the choice to invest in various elements of critical natural capital always also implies value-based choices, beyond technical considerations of efficiency and technical constraints only. In particular, the definition of the critical level of natural capital will depend both on the scientific understanding of the complex dynamics of coupled socio-ecological systems and on the broader social debate on value-laden goals and objectives. This complex interdependence between discussions on normative values and factual knowledge is one of the reasons why work in environmental ethics should be conducted in close dialogue with socio-economic analysis and the environmental sciences, amongst others. It also reinforces the argument 
made by most sustainability scholars that the three requirements of sustainability science (the better understanding of the ethical dimension, the complex systems' analysis of coupled socio-ecological systems and the transformational agenda) should be satisfied together. Therefore, these questions for operationalizing strong sustainability should be considered as research questions and not just as implementation tasks for people outside sustainability science.

In his discussion of sustainability, Rutger Claassen gives some interesting illustrations of contemporary debates which can illustrate this latter point, by using one possible technical measure of critical capital amongst others, which is the notion of an individual person's ecological footprint (Claassen, 2011). The ecological footprint measures all the resources that an individual uses (from fish and meat to paper and petrol) in terms of the hectares of biologically productive land and sea area necessary to supply these resources, and to assimilate associated waste. Using the model of the ecological footprint, it is possible to estimate how much of the earth (or how many planets earth) it would take to support humanity, if everybody followed a given lifestyle. With the current world population, 1.8 hectares are available for each individual human being. At present, the average individual ecological footprint for the Belgian lifestyle is 8 hectares, while the average footprint of the Chinese lifestyle is 2.2 hectares and the Indian lifestyle 0.9 hectares (National Footprint Accounts, 2012). Altogether, in the current situation, this leads to an average actual use of 2.7 hectares per human being for 2007, which is clearly an unsustainable situation. In other words, in 2007, humanity's total ecological footprint was estimated at 1.5 planets earth; that is, humanity uses ecological services 1.5 times as quickly as the planet can renew them.

A first ethical question to be addressed, in the analysis of ecological footprint data, is to know what species deserve to be included in the measure of present and future needs of natural capital. The 1.8 hectares mentioned above is based only on the use of the planet for direct use by human beings. However, most sustainability scholars would argue for the need to include a certain level of natural capital for other species as well, in order to maintain a certain level of biological diversity on earth. Such an inclusion of other species also has a cost: in one study, Jones and Jacobs (2007) showed that, in such a modified scenario, the available hectares per person would decrease to 1.6 per person (from 1.8 in a human-needs only scenario). The question of the basis on which such a "gift" is justified is intensely debated. Some think that the anthropocentric ethics of the original ecological footprint analysis is unacceptable and that we need to adopt an eco-centric perspective, which also values nature for its intrinsic worth (Sober, 1986; Desjardins, 2005). Others argue that human beings are dependent on the 
resilience of ecosystems - which is their capacity to regenerate after severe disturbances and shocks - and biodiversity is of crucial importance to such resilience. Therefore, there is no need to adopt an eco-centric perspective to include such indirect and long-term usefulness of biodiversity for human beings in the calculus of the ecological footprint. Still another position shows the importance of nature conservation as a component of cultural capital, as nature also has a sacred or an aesthetical value for various communities and individuals and therefore also plays an important role in their aspirations to a meaningful life.

A second ethical question is how far we need to factor in the growth in consumption in developing and emerging economies. Indeed, even if the ecological footprint in the rich countries needs to decrease, it seems fair to admit that the developing and emerging economies have the right to further develop and to increase their own ecological footprint from the current average of 0.5 to 1.5 hectares per person. Such a perspective leads us to consider that the natural resources are a kind of common heritage, which should be equally shared amongst all. The latter position, however, leads to complex political questions. The calculus of the ecological footprint in Belgium, for example, also includes the use by an average Belgian lifestyle of hectares outside Belgian in developing countries to satisfy his or her own needs (such as hectares of rainforest cut down to produce soya for animal feed in Belgium), both in terms of the direct use of resources and of the assimilation of associated wastes. Therefore, the issue of the limits on the earth's resources cannot be considered independently of issues of global equity in benefiting from these resources.

The ecological footprint indicator, as any indicator, has many shortcomings and needs to be considered together with other possible approaches to operationalizing the ethics of strong sustainability. However, what these ethical questions highlighted by Rutger Claassen (2011) show is the need to move beyond the technical and expert-based calculus of critical thresholds of natural capital only. Indeed, in operationalizing the ethics of strong sustainability, sustainability research also needs to address the various context-specific value-laden goals and objectives that play a role in the practical definition of certain criteria of sustainability for a given community, city, geographical region or country. In short, to operationalize the ethical dimension of sustainability science there is a need to clarify the ethical debates on specific objectives for reaching sustainability in a given context, in combination with the building of common ground on general ethical frameworks and indicators. Typical tasks to be fulfilled in clarifying the ethical foundation of sustainability science and in contributing to effective policy are, for example (see Baumgaertner and Quaas, 2010): 
- the development of specific notions of efficiency and justice for socio-ecological systems, and the corresponding ethics that explicitly deals with the long-term future, which is inherently uncertain and, beyond that, to a significant extent, in principle unknowable;

- the clarification of the relationships among the different value-laden goals of various sustainability stakeholders and the identification of potential conflicts and trade-offs;

- the development of operational qualitative and quantitative indicators for the value-laden goals, and the determination of adequate targets and tolerable windows for the indicators for specific contexts.

\subsection{AN INTEGRATED PERSPECTIVE ON SOCIO- ECOLOGICAL SYSTEMS}

Several characteristics of persistent problems of unsustainability present serious challenges for scientific research. As Jaeger (2011) points out: "for each of the different problems (climate change, land degradation, biodiversity loss, etc.) or problem sector (agriculture, energy, transport, etc.) the symptoms of unsustainability mask deeper underlying problems in our societal structures and institutions". Thus, as Rotmans et al. (2001) also stresses, these problems cannot be solved in isolation. According to their analyses, the complexity arises because of the multiple and interacting drivers of change (for example agriculture requires land, water and energy), the interactions within the earth system (for example between the atmosphere and the oceans or between climate and vegetation), the interactions between levels of scale, time delays in responses of ecosystems to external shocks and because of the massive complexities of human consumption and production systems. Further, an important feature of the coupled socio-ecological system, which results from the complexity, is the presence of different types of uncertainty, ranging from simply technicalstatistical, to methodological (choice of methods) and epistemological levels (irremediable uncertainty, irreducible lack of knowledge). The complexities and uncertainties, together with the fact that there are multiple stakeholders, mean that normal scientific research projects are ill equipped to deal with persistent problems of unsustainability.

\subsubsection{Navigating Complex Socio-ecological Interactions}

The multiple-scale and multi-faceted features of sustainability problems clearly challenge the effectiveness of the analysis of socio-ecological systems. Arguably, the traditional scientific approach, which tends to build 
systems as aggregates of elements which, for the purposes of analysis, can ignore the integrated or emergent outcomes of their interconnection, is not appropriate for the field of sustainable development. However, as discussed above, most current scientific thinking about natural resources and sustainability is still driven by a "frontier economics" mentality, where biophysical limits are axiomatically assumed not to exist, or, at least, are considered as not imposing particularly important limits on the system under consideration. Further, all too often, the analysis leads to the proposition of optimal solutions instead of suggesting a set of tools for improved diagnostics and adaptive learning by actors and policy makers interacting and operating in complex socio-ecological systems.

Current science seems to work well for problems which are compartmentalized, but does not perform well in providing answers to problems that are systemic, interdependent, multi-scale (temporal and spatial) and multifaceted (i.e. with economic, political and environmental facets). Indeed, the presumption that scholars can generate simple, predictive models of coupled socio-ecological systems and deduce general solutions has led to a track record of repeated and often dramatic failures (Ostrom et al., 2007). Higgs (1996, p. 247) for example outlines how efforts to turn the regulation of the Washington salmon fishery entirely over to the state government, a frequently recommended cure-all, generated "a legal and economic horror story" that reduced the productivity of the fishery to a small fraction of what it was at the turn of the twentieth century. Bacho (2005) documents how the panacea of decentralization, as implemented in a multi-ethnic district of Ghana, generated extensive ethnic conflict. Gelcich et al. (2006) report how imposing a blueprint co-management system on a traditional lottery system for managing a marine ecosystem weakened the level of trust in a community and intensified conflict. Von Weizsaecker et al. (2005) challenge the view that privatization is always the best option for delivering public services and present 50 case studies on best-case and worst-case experience of efforts to privatize water, transport and energy.

Advocates of cure-alls or panaceas make two false assumptions (Ostrom et al., 2007): (i) all problems, whether they are different challenges within a single resource system or across a diverse set of resource systems, are similar enough to be represented by a small class of formal models; and (ii) the set of preferences, the possible roles of information, and individual perceptions and reactions are assumed to be the same as those found in developed Western market economies. To move beyond panaceas and build a solid field of sustainability science, a more fruitful approach is to recognize that complex systems cannot be separated into linear independent parts, but are only partially decomposable into their structure (Ostrom, 2007). Simon (2000, p. 753) describes nearly decomposable systems as 
being "arranged in levels, the elements at each lower level being subdivisions of the elements above ... Multi-celled organisms are composed of organs, organs of tissues, tissues of cells". One consequence of a complex systems' approach is the need to specify at what level and in what part of the system policies apply. Indeed, policies can be explored in one part of a system without imposing uniform solutions on the larger system that might lead to a large-scale collapse. Second, it is essential for scholars to recognize that combining variables, for instance $\mathrm{A}, \mathrm{B}$ and $\mathrm{C}$, can lead to a system with emergent properties that differ substantially from combining two of the original variables with a different one, say A, B and D.

\subsubsection{Building Integrated Frameworks of Analysis}

Sustainability scholars have developed a set of tools and practices to address the complex dynamic interaction between nature and society in an integrated way, without having recourse to the reductionist fallacies described above. For example, in the context of political science, Ostrom (2007) proposed an analytic framework for the comparative institutional analysis of coupled socio-ecological systems consisting of a resource system (for example a fishery, lake, grazing area), resource units generated by that system (for example fish, water, fodder), the users of that system and the governance system, where all these components and their interactions are related to other ecosystems and constrained by social, economic and political settings. Another important framework was proposed by Herman Daly in ecological economics (Daly, 2005). This framework is based on a nested hierarchical model in which the socio-economic system is a sub-system of the overall biophysical system. In addition, Daly's framework emphasizes the feedback loops amongst materials, energy resources, technology, information flows and production processes underlying economic activity.

Needless to say, there will never be one generic framework useful for all research agendas. However, an important point to underline is that each single discipline will have to revise and adapt its own basic framework principles in order to address the sustainability problems in an integrated way (as Ostrom and Daly started developing a new framework for complex systems analysis in political science and economics respectively).

Analysing the multiple processes occurring in complex, nested, socioecological systems is far more challenging than recommending a favourite cure-all solution. In a similar way to other strategic sciences such as medicine or engineering, sustainability science aims to find diverse solutions to complex problem situations, based on initial diagnosis, deeper analysis, continuous monitoring of various indicators and systematic learning from 
failures. The insistence of sustainability scholars on adopting a diagnostic and iterative approach for the study of the coupled dynamics between ecological and socio-ecological systems again emphasizes that sustainability science is neither a purely descriptive-analytical science, nor is it a purely normative endeavour, but an interactive and iterative learning process that combines elements of both. It is what has been called "strategic science" (European Commission, 2009) or "relevant science" (Baumgaertner and Quaas, 2010, p. 447; see also the discussion above in the introduction to Chapter 2).

Taking an integrated view of socio-ecological systems, in which scarce resources are used over a long time and under conditions of uncertainty, leads to a set of specific and genuine sustainability science research questions, contributing to the core aim of achieving the policy goal of sustainability in its ecological, economic, social, cultural and governance dimensions. Examples of research tasks that are based on such an integrated perspective on socio-ecological systems are (adapted from Baumgaertner and Quaas (2010) and Kajikawa's (2008) surveys of the literature):

- analysis of the interaction between physical (for example biophysical, energy-matter) and socio-economic (for example based on monetary and non-monetary values) variables in socio-ecological systems, for example in ecological economic modelling and analysis;

- analysis of dynamic socio-ecological systems, taking into account feedback and the emergence of system properties such as thresholds, critical loads, and limited resilience in social, environmental and coupled socio-ecological systems;

- analysis of different types, degrees and patterns of uncertainty in our understanding of coupled socio-ecological systems;

- analysis of conditions and mechanisms that affect the social, economic and political stability of socio-ecological systems, and analysis of stability patterns, vulnerability and systemic risks;

- analysis of conditions and mechanisms that affect the transformability of socio-ecological systems, and the analysis of transition pathways towards sustainability.

\subsection{A TRANSDISCIPLINARY RESEARCH ORGANIZATION FOR SUSTAINABILITY SCIENCE}

Dissatisfaction with the shortcomings of current methods of producing and validating scientific knowledge has given rise to various proposals for 
reconsidering and renewing the epistemological and social foundations of science. As part of this new "social contract for science" (Demeritt, 2000; Gibbons, 1999), not only would science "speak truth to power", but society would also "speak back to science" in identifying relevant topics and research priorities, questioning the relevance of specific methodologies and assumptions, validating the results in terms of their social robustness, and making normative commitments explicit.

\subsubsection{Addressing Situations of Irreducible Uncertainty, Multiple Values and High Stakes}

Silvio Funtowicz and Jerome Ravetz (1993) have attempted to better specify the terms of this social contract (and the contexts in which it is particularly needed). According to them, the traditional methodology of modern science, based on disciplinary and value-neutral scientific expertise, is generally suitable for so-called "normal" contexts. In such contexts the elements of human and biophysical systems can be validly separated for research purposes, uncertainty is relatively low and natural resource limitations are not relevant. In contrast, when uncertainty is high and when the systemic interconnection of various systems and the resource constraints cannot be ignored, a different mode of organization of scientific research is needed, based on transdisciplinary collaboration between scientific and sustainability stakeholders' expertise. In these socalled post-normal contexts, the description of facts through a unique methodological lens and the unidirectional path from research to policy conclusions are likely to prove inappropriate.

In their seminal article on "Science for the post-normal age", Silvio Funtowicz and Jerome Ravetz (1993) identified two key challenges for science in post-normal contexts: the challenge of dealing with uncertainty and the generalization of extended peer review for improved quality management of the scientific process. According to their analysis:

now that the policy issues of risk and the environment present the most urgent problems for science, uncertainty and quality are moving in from the periphery, one might say the shadows, of scientific methodology, to become the central, integrating concepts. Hitherto they have been kept at the margin of the understanding of science, for laypersons and scientists alike. A new role for scientists will involve the management of these crucial uncertainties; therein lays the task of quality assurance of the scientific information provided for policy decisions (Funtowicz and Ravetz, 1993, p. 742).

In response to these and other calls for a "new social contract" for science and the need for extended peer review, a large body of literature on 
transdisciplinary, community-based, interactive and participatory research approaches as well as empirical projects has been generated (Lang et al., 2012). Transdisciplinarity in particular has been at the heart of these emergent practices of sustainability. Although an open and still evolving concept, there is a growing consensus that the key features of transdisciplinary research are the integration of scientific and various extra-scientific expertise from the relevant stakeholder communities and the linking of scientific problems with societal problems (Jahn et al., 2012). More specifically, sustainability scholars define transdisciplinary research as a "reflexive, integrative, method-driven scientific principle aiming at the solution or transition of societal problems, and concurrently of related scientific problems, by differentiating and integrating knowledge from various scientific and societal bodies of knowledge" (Jahn et al., 2012, pp. 26-7). For example, people directly affected by an environmental problem will have a keener awareness of its symptoms, and a more pressing concern with the quality of official reassurances, than those in other roles (Funtowicz and Ravetz, 1993). Thus their societal body of knowledge can function in an analogous way to that of professional colleagues in the peerreview or refereeing process in traditional science. An historical example of the possible contribution of such extended expertise is the use of the knowledge of the inhabitants of the city of Lyme, whose lay expertise led to the recognition of a new disease, later called "Lyme's disease", which had not previously been recognized as being a new disease by the conventional scientific experts. More recent examples of extended expertise will be discussed in Chapter 4 below.

\subsubsection{An Illustrative Model of a Transdisciplinary Research Process}

The lack of experience with transdisciplinary research practice, when dealing with problems of strong sustainability, has led to a long history of failure of research projects that has been well documented in the literature (Lang et al., 2012, pp. 33-4). Familiar problems are the lack of transferability of the scientific research results into practice, or even the misuse of results to legitimate unintended actions; the lack of integration across knowledge types, organizational structures, communicative styles, or technical aspects; and the underrepresentation of relevant issues in the definition of the problems to be addressed.

To cope with these and other issues, transdisciplinary researchers propose an "interface practice" between a societal practice of social problem solving and a scientific practice of interdisciplinary analysis. The interaction between these two practices is typically organized in three main components (Lang et al., 2012, p. 27; see also Figure 2.1), including: (a) the collaborative framing of the problem and the building of a collaborative research team composed of scientific and non-scientific experts on the 


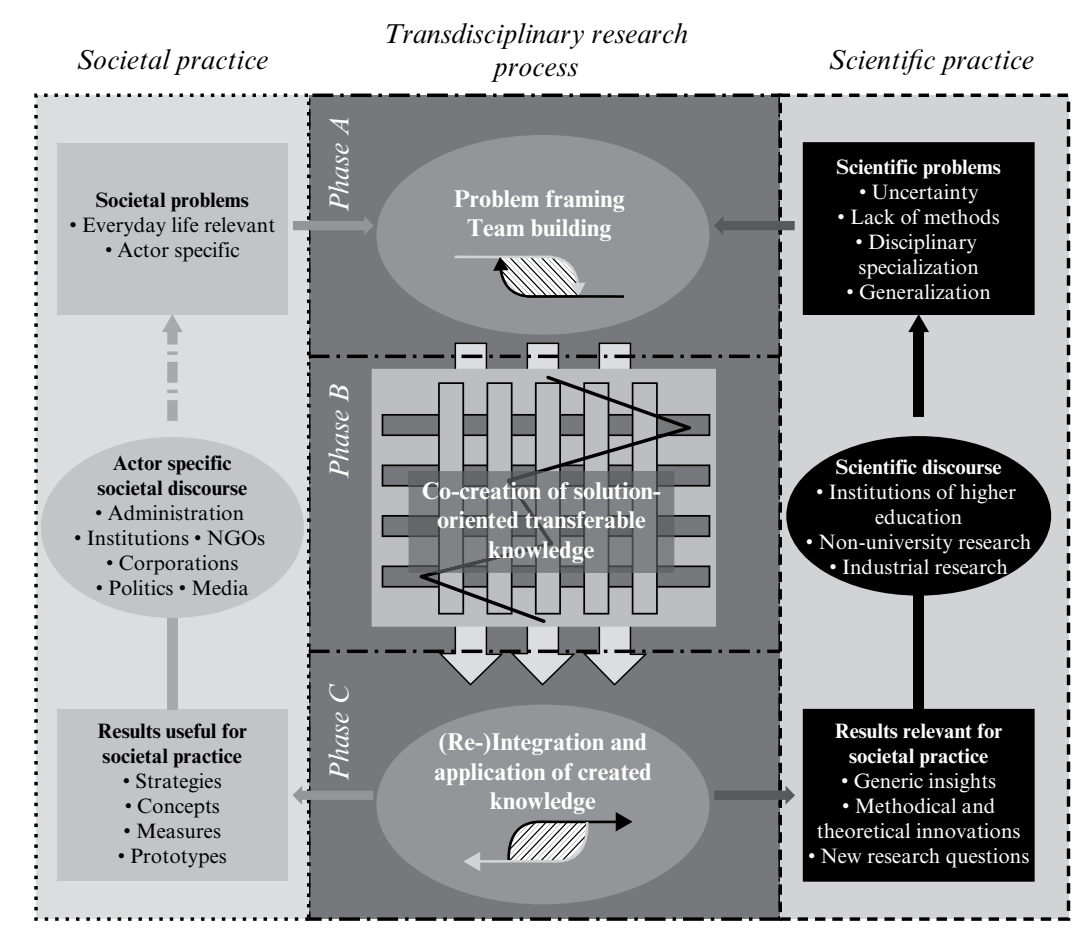

Source: Lang et al. (2012, p. 28).

Figure 2.1 Conceptual model of an ideal-typical transdisciplinary research process

relevant scientific and societal bodies of knowledge; (b) joint knowledge production through collaborative scientific research; and (c) the integration of scientific results into societal practice (for example as a diagnostic tool that can be used by the actors concerned) and in the scientific practice (for example, by learning from system failures that were discovered in the collaborative research project, but not initially predicted by the formal models). This schematic representation of the research cycle has been further elaborated to include the many iterations of this process in practical research programmes. This and other well-tested examples of research design clearly show the possibility of integrating conventional scientific disciplinary expertise into a mutual learning process among researchers and other actors, in a broadened organization of the quality management of the overall research cycle. 